\title{
Solitons of axion-dilaton gravity
}

\author{
Ioannis Bakas* \\ Theory Division, European Organization for Nuclear Research (CERN), 1211 Geneva 23, Switzerland \\ and Department of Physics, University of Patras, 26110 Patras, Greece ${ }^{\dagger}$
}

(Received 1 July 1996)

\begin{abstract}
We use soliton techniques of the two-dimensional reduced $\beta$-function equations to obtain nontrivial string backgrounds from flat space. These solutions are characterized by two integers $(n, m)$ referring to the soliton numbers of the metric and axion-dilaton sectors, respectively. We show that the Nappi-Witten universe associated with the $\mathrm{SL}(2) \times \mathrm{SU}(2) / \mathrm{SO}(1,1) \times \mathrm{U}(1)$ CFT coset arises as a (1,1) soliton in this fashion for certain values of the moduli parameters, while for other values of the soliton moduli we arrive at the $\operatorname{SL}(2) / \operatorname{SO}(1,1)$ $\times \mathrm{SO}(1,1)^{2}$ background. Ordinary four-dimensional black holes arise as two-dimensional $(2,0)$ solitons, while the Euclidean wormhole background is described as a $(0,2)$ soliton on flat space. The soliton transformations correspond to specific elements of the string Geroch group. These could be used as a starting point for exploring the role of $U$ dualities in string compactifications to two dimensions. [S0556-2821(96)03422-4]

PACS number(s): 11.25.Sq, 02.20.Tw, 04.60.Kz
\end{abstract}

\section{INTRODUCTION}

Duality symmetries in string theory arise as discrete remnants of continuous groups of transformations of the lowest order effective theory. These symmetries have received a lot of attention, as they can also provide nonperturbative information about string theory. The most common examples are $T$ and $S$ dualities, but it has also become clear recently that $U$ dualities can be successfully used to explore various generalized equivalences among superstrings $[1,2]$.

Dimensional reduction offers the possibility to intertwine the $T$ and $S$ moduli, and hence construct large groups of solution-generating symmetries in three and two dimensions. For example, the reduction from four to three dimensions gives rise to an $\mathrm{O}(2,2)$ group [3], while the reduction from four to two dimensions leads to an infinite dimensional group of the lowest order effective theory, the current group $\hat{\mathrm{O}}(2,2)[4]$. These results can be regarded as straightforward generalization of similar structures found by Geroch in the space of solutions of vacuum Einstein equations with one or two commuting isometries [5], but now they also include apart from the metric $G_{\mu \nu}$ the antisymmetric tensor field $B_{\mu \nu}$ and the dilaton $\Phi$. The coset space structure of the scalar fields in various dimensionally reduced supergravity theories was known before (see, for instance, [6-8] and references therein). More recent is the realization that $T$ and $S$ dualities are embedded in the corresponding continuous hidden symmetry groups. In a heterotic string context it means that the reduction from ten to three dimensions gives rise to an $\mathrm{O}(8,24)$ group $[7,9]$, while the reduction from ten to two dimensions leads to $\hat{\mathrm{O}}(8,24)[10,11]$. It is then natural to expect that the two-dimensional (2D) sector of string theory will be quite rich in symmetry, having as $U$ duality an appropriately chosen discrete subgroup of the underlying string

\footnotetext{
*Electronic addresses: BAKAS@SURYA11.CERN.CH, BAKAS@NXTH04.CERN.CH

†Permanent address.
}

Geroch current group. Up to this day, however, very little progress has been made in this particular direction, since proving the conjectured $U$ dualities and understanding their action on the full spectrum of superstring models based on these effectively 2D backgrounds turns into a difficult problem.

In this paper we consider string models with target space $M_{4} \times K$, where $M_{4}$ is a $4 \mathrm{D}$ spacetime with signature -+++ and $K$ is some internal space, which is usually represented by a conformal field theory (CFT), so that the total central charge is critical. We focus on cosmological backgrounds $M_{4}$ with nontrivial $G_{\mu \nu}, B_{\mu \nu}$, and $\Phi$ that arise as solutions of the lowest order effective theory:

$$
S_{\mathrm{eff}}=\int_{M_{4}} d^{4} X \sqrt{-\operatorname{det} G}\left[R-2\left(\nabla_{\mu} \Phi\right)^{2}-\frac{1}{12} e^{-4 \Phi} H_{\mu \nu \rho}^{2}\right]
$$

Here, the theory is defined directly in the Einstein frame, which is related to the $\sigma$-model frame by $G_{\mu \nu}^{(\sigma)}=e^{2 \Phi} G_{\mu \nu}$, and the effective cosmological constant is taken as zero. It will be convenient for later use to trade $B_{\mu \nu}$ with the axion field $b$, which is consistently defined in the Einstein frame as

$$
\partial_{\mu} b=\frac{1}{6} e^{-4 \Phi} \sqrt{-\operatorname{det} G} \epsilon_{\mu}{ }^{\nu \rho \sigma} H_{\nu \rho \sigma}
$$

$H_{\mu \nu \rho}$ is the field strength of $B_{\mu \nu}$ and $\epsilon_{0123}=1$. In $M_{4}$ with signature -+++ we may further define the complex conjugate fields $S_{ \pm}=b \pm i e^{-2 \Phi}$, which provide the natural variables of the $S$ moduli. Later, we will also consider string backgrounds with Euclidean signature ++++ .

There is a very limited number of exact CFT backgrounds, which to lowest order in $\alpha^{\prime}$ provide solutions of Eq. (1.1). The most characteristic examples of this type are the two pairs of WZW coset models:

$$
\frac{\mathrm{SL}(2) \times \mathrm{SU}(2)}{\mathrm{SO}(1,1) \times \mathrm{U}(1)}, \frac{\mathrm{SL}(2)}{\mathrm{SO}(1,1)} \times \mathrm{SO}(1,1)^{2},
$$




$$
\frac{\mathrm{SL}(2)}{\mathrm{SO}(1,1)} \times \frac{\mathrm{SU}(2)}{\mathrm{U}(1)}, \quad \mathrm{SL}(2) \times \mathrm{SO}(1,1) .
$$

The first model in Eq. (1.3) depends on a free parameter that defines the gauging of the coset, and it is particularly interesting in string cosmology as it describes a closed inhomogeneous expanding and recollapsing universe [12] (see also [13] for some earlier ideas). The other three models are the Lorentzian counterparts obtained by analytic continuation of the $D=4, \quad \hat{c}=4, \quad N=4$ superconformal backgrounds $\mathrm{C}^{(4)}=\mathrm{SU}(2) / \mathrm{U}(1) \times \mathrm{U}(1)^{2}, \quad \Delta^{(4)}=\mathrm{SL}(2) / \mathrm{U}(1) \times \mathrm{SU}(2) /$ $U(1)$ and $W^{(4)}=\mathrm{SU}(2) \times \mathrm{U}(1)$ (the throat of a wormhole), respectively, with appropriately chosen background charges [14-16]. All these models exhibit two commuting Killing isometries. It has been established with the aid of $\mathrm{O}(2,2)$ transformations that the first model in Eq. (1.3) is related to the first model in Eq. (1.4) [17], and similarly the other two models of the series are $T$ dual to each other $[15,18]$.

Our contribution is to connect the two gravitational backgrounds associated with the Nappi-Witten universe [12] $\mathrm{SL}(2) \times \mathrm{SU}(2) / \mathrm{SO}(1,1) \times \mathrm{U}(1) \quad$ and $\quad \mathrm{SL}(2) / \mathrm{SO}(1,1)$ $\times \mathrm{SO}(1,1)^{2}$ to the trivial flat space background $F^{(4)}$ with zero $B_{\mu \nu}$ and $\Phi$, by considering a specially chosen sixdimensional moduli space of backgrounds within the entire set of solutions of Eq. (1.1) with two commuting Killing isometries. This is technically achieved by performing first the $2 \mathrm{D}$ reduction of the effective theory (1.1), and then employing solitonic constructions that are available for the resulting integrable system of equations (both for the metric and the axion-dilaton sectors). As it turns out, the simplest $(1,1)$ soliton configuration on $F^{(4)}$, or more precisely its $T$-dual face, will be sufficient to describe the semiclassical backgrounds of these two coset models as 2D solitons for appropriate choices of the six moduli parameters. The solitonic dressing of (the dual of) $F^{(4)}$ in this paper is analogous to the solitonic dressing of Kasner-type metrics that were studied by Belinski and Sakharov in the context of general relativity many years ago [19]. In the context of pure gravity these authors gave a very interesting derivation of 4D black holes as 2D double soliton solutions on flat space. Further work has also appeared in the literature, which describes the physically very interesting situation of two colliding gravitational plane waves in terms of 2D solitons [20]. Given the wide applicability of these methods, it is also natural to consider the explicit form of solitons in axion-dilaton gravity and attempt a reinterpretation of known solutions, in particular those that correspond to exact CFT backgrounds, in this context.

It is interesting to note that the soliton dressing of a given configuration corresponds to a specific choice of finite group element of the Geroch group (see, for instance, [21]), and hence in the string context we have found the way to generate the two exact CFT backgrounds (1.3) from flat space by $U$ duality (viewed as a continuous group of transformations at this point). In a sense one may then say that the dualities create a universe, the Nappi-Witten cosmological solution in this case. We will also consider Euclidean string backgrounds and show for example, that the wormhole solution, which is an axionic instanton of the 10D heterotic theory, arises as a $2 \mathrm{D}(0,2)$ soliton on flat space. Certainly, many more connections can be made between different string backgrounds using the inverse-scattering method of the $2 \mathrm{D}$ reduced sector, and we hope to return to them in the near future.

In Sec. II we briefly discuss the dimensionally reduced string background equations and outline the construction of soliton solutions using the integrability of the resulting $2 \mathrm{D}$ $\sigma$ models. In Sec. III we construct the most general $(1,1)$ soliton solution on a $T$-dual face of Minkowski space and determine the choice of moduli parameters that correspond to the CFT backgrounds $\mathrm{SL}(2) \times \mathrm{SU}(2) / \mathrm{SO}(1,1) \times \mathrm{U}(1)$ and $\mathrm{SL}(2) / \mathrm{SO}(1,1) \times \mathrm{SO}(1,1)^{2}$. In Sec. IV we describe the ordinary $4 \mathrm{D}$ black holes as $2 \mathrm{D}(2,0)$ solitons on flat space, where the soliton moduli correspond to the mass, rotation, and Newman-Unti-Tamburino (NUT) parameters of the most general stationary axisymmetric solution. In Sec. V the Euclidean wormhole background is interpreted as a $2 \mathrm{D}(0,2)$ soliton in the same context. Section VI contains our conclusions and some directions for further work on the subject. We argue that the present results could be most importantly used as starting point for exploring the role of $U$ dualities in compactifications of string theory to two dimensions.

\section{THE REDUCED THEORY AND ITS SOLITONS}

The effective theory (1.1) describes the coupling of an ordinary $\mathrm{SL}(2) / \mathrm{U}(1) \sigma$ model to $4 \mathrm{D}$ gravity, which is manifest in the axion-dilaton formulation using the field variables $S_{ \pm}=b \pm i e^{-2 \Phi}$. This axion-dilaton $\sigma$ model has Lorentzian signature if $M_{4}$ is Euclidean, but in the case of interest here, where $M_{4}$ is Lorentzian, the SL(2)/U(1) $\sigma$ model is Euclidean. Hence, it is convenient to parametrize the axion-dilaton sector by the symmetric matrix

$$
\lambda=e^{2 \Phi}\left(\begin{array}{ll}
1 & b \\
b & b^{2}+e^{-4 \Phi}
\end{array}\right)
$$

so that $\operatorname{det} \lambda=1$.

Following [4] (and references therein) we consider gravitational string backgrounds of cosmological-type with two commuting Killing isometries, so that the target space metric is restricted by the Ansatz.

$$
\begin{aligned}
d s^{2}= & f\left(X^{0}, X^{1}\right)\left[-\left(d X^{0}\right)^{2}+\left(d X^{1}\right)^{2}\right] \\
& +g_{A B}\left(X^{0}, X^{1}\right) d X^{A} d X^{B}, \quad A, B=2,3,
\end{aligned}
$$

and also $b\left(X^{0}, X^{1}\right), \Phi\left(X^{0}, X^{1}\right)$. For notational convenience we introduce the light-cone coordinates

$$
\eta=\frac{1}{2}\left(X^{0}-X^{1}\right), \quad \xi=\frac{1}{2}\left(X^{0}+X^{1}\right) .
$$

Then, using the defining relations (1.2) this Ansatz amounts to choosing special backgrounds with only $B_{23} \neq 0$, in which case the axion equations simplify to

$$
\partial_{\xi} b=\frac{e^{-4 \Phi}}{\sqrt{\operatorname{det} g}} \partial_{\xi} B_{23}, \quad \partial_{\eta} b=-\frac{e^{-4 \Phi}}{\sqrt{\operatorname{det} g}} \partial_{\eta} B_{23} .
$$


This class of backgrounds will be quite sufficient for the present purposes of our work.

The reduced string background equations that follow from Eq. (1.1) read as follows in the Einstein frame:

$$
\begin{aligned}
& \partial_{\eta}\left(\sqrt{\operatorname{det} g} g^{-1} \partial_{\xi} g\right)+\partial_{\xi}\left(\sqrt{\operatorname{det} g} g^{-1} \partial_{\eta} g\right)=0, \\
& \partial_{\eta}\left(\sqrt{\operatorname{det} g} \lambda^{-1} \partial_{\xi} \lambda\right)+\partial_{\xi}\left(\sqrt{\operatorname{det} g} \lambda^{-1} \partial_{\eta} \lambda\right)=0 .
\end{aligned}
$$

They are two essentially decoupled SL(2)/U(1) 2D $\sigma$ models of Ernst-type (both having Euclidean signature), one for the metric sector $g$ and the other for the axion-dilaton $\lambda$. Since $\sqrt{\operatorname{det} g}$ satisfies the 2D wave equation $\partial_{\eta} \partial_{\xi} \sqrt{\operatorname{det} g}=0$, we may choose, without loss of generality,

$$
X^{0}=\sqrt{\operatorname{det} g} \equiv \alpha, \quad X^{1}=\beta
$$

for the corresponding pair of its conjugate solutions. From now on we assume the special choice of coordinates (2.7), using $\alpha=\sqrt{\operatorname{det} g}$ and $\beta$ instead of $X^{0}$ and $X^{1}$ in Eq. (2.2), and reserve the notation $X^{0}, X^{1}$ for more general coordinate systems. Sometimes, we will also denote the remaining two coordinates by $z$ and $w$ instead of $X^{2}$ and $X^{3}$, in order to make more uniform our presentation in the following sections.

We recall that the differential equations for the conformal factor $f$ are linear of first order,

$$
\begin{aligned}
& \partial_{\xi}(\ln f)=-\frac{1}{\alpha}+\frac{\alpha}{4} \operatorname{Tr}\left[\left(g^{-1} \partial_{\xi} g\right)^{2}+\left(\lambda^{-1} \partial_{\xi} \lambda\right)^{2}\right], \\
& \partial_{\eta}(\ln f)=-\frac{1}{\alpha}+\frac{\alpha}{4} \operatorname{Tr}\left[\left(g^{-1} \partial_{\eta} g\right)^{2}+\left(\lambda^{-1} \partial_{\eta} \lambda\right)^{2}\right],
\end{aligned}
$$

and so, once a solution $(g, \lambda)$ of the two Ernst $\sigma$ models is known, $f$ can be simply determined integrating by quadratures. All these calculations are performed in the Einstein frame, where the decoupling of Eqs. (2.5) and (2.6) takes place, but the results can be easily translated in the $\sigma$-model frame of string theory.

The nonlinear $\sigma$ models of the 2D reduced theory are known to be integrable, and it is precisely this property that is responsible for having an infinite-dimensional symmetry group, the (string) Geroch group, acting on the space of classical solutions. Because of integrability the 2D $\sigma$ models admit soliton solutions, which can be constructed explicitly on any given background that acts as a seed for the solitons. On any string background we may actually construct a whole series of solitonic excitations $(n, m)$, where $n$ and $m$ denote the soliton numbers of the $g$ and $\lambda$ sectors, respectively. Here, we briefly review the essential ingredients of the soliton technique for the Ernst $\sigma$ model using only the metric sector of the theory, but the construction is exactly the same for the axion-dilaton sector since $\sqrt{\operatorname{det} g} \lambda$ satisfies the same equation (2.6) as $\lambda$. The new solutions that arise in this fashion are solitons in the 2D sense, and although 4D string backgrounds can be reconstructed from the $(n, m)$ data, the resulting configurations are not necessarily solitons of the 4D world saturating the Bogomol'ny bound, and thus they are generically quantum-mechanically unstable. Next, we revisit the soliton framework of Belinski-Sakharov [19], because their technique is not widely known to string theorists.

Consider the following linear system of $(2 \times 2)$-matrix differential equations:

$$
D_{1} \Psi=\frac{A}{l-\alpha} \Psi, \quad D_{2} \Psi=\frac{B}{l+\alpha} \Psi,
$$

where $\Psi(\eta, \xi ; l)$ is a complex matrix function depending on a spectral parameter $l$ that takes values in the whole complex plane, and

$$
A=-\alpha \partial_{\xi} g g^{-1}, \quad B=\alpha \partial_{\eta} g g^{-1} .
$$

Also, the differential operators are

$$
D_{1}=\partial_{\xi}-2 \frac{l}{l-\alpha} \partial_{l}, \quad D_{2}=\partial_{\eta}+2 \frac{l}{l+\alpha} \partial_{l}
$$

and clearly they commute, $\left[D_{1}, D_{2}\right]=0$. The system (2.10) is compatible provided that $g$ satisfies the Ernst equation (2.5) for $\sqrt{\operatorname{det} g}=\alpha$. We actually assume

$$
\Psi(\eta, \xi ; l=0)=g(\eta, \xi),
$$

and so $\Psi$ can be regarded as a suitable generalization of $g$ with spectral parameter.

Let $g_{0}(\eta, \xi)$ be a known solution and let $\Psi_{0}(\eta, \xi ; l)$ be the corresponding solution of the linear system (2.10). If we assume that other solutions of $g$ exist such that

$$
\Psi(l)=\chi(l) \Psi_{0}(l),
$$

then $\chi(\eta, \xi ; l)$ has to satisfy the system of equations

$$
D_{1} \chi=\frac{1}{l-\alpha}\left(A \chi-\chi A_{0}\right), \quad D_{2} \chi=\frac{1}{l+\alpha}\left(B \chi-\chi B_{0}\right),
$$

where $A_{0}, B_{0}$ are the currents (2.11) of a seed metric $g_{0}$. If we manage to find an appropriate $\chi(l)$, then according to Eqs. (2.13) and (2.14), a new solution will be obtained

$$
g(\eta, \xi)=\chi(l=0) g_{0} .
$$

There are a few technical assumptions on $\chi$, namely, the reality condition on the real $l$ line, $\bar{\chi}(\bar{l})=\chi(l)$, and $\chi(\infty)=1$.

The $n$-soliton excitations of a given seed background $g_{0}$ are very special in that $\chi$ has a simple pole structure in the complex $l$ plane

$$
\chi(\eta, \xi ; l)=1+\sum_{k=1}^{n} \frac{R_{k}(\eta, \xi)}{l-\mu_{k}(\eta, \xi)} .
$$

The residue and pole functions can be determined substituting Eq. (2.17) in Eq. (2.15) and start comparing the pole structure on the left- and right-hand sides. The details are rather lengthy and we skip them here. We only give the final result that will be used later for explicit computations. The poles are roots of the algebraic equation

$$
\mu_{k}^{2}+2\left(\beta-C_{0}^{(k)}\right) \mu_{k}+\alpha^{2}=0,
$$


where $C_{0}^{(k)}$ are arbitrary numerical constants (moduli), and $\mu_{k}$ satisfy the differential equations in $\eta, \xi$ :

$$
\partial_{\eta} \mu_{k}=\frac{2 \mu_{k}}{\alpha+\mu_{k}}, \quad \partial_{\xi} \mu_{k}=\frac{2 \mu_{k}}{\alpha-\mu_{k}} .
$$

The residue matrices $R_{k}$ are degenerate having the component form

$$
\left(R_{k}\right)_{A B}=N_{A}^{(k)} M_{B}^{(k)},
$$

where the two-component vector $M^{(k)}$ is given using the inverse of $\Psi_{0}$ at $l=\mu_{k}$,

$$
M_{B}^{(k)}=\sum_{A} C_{A}^{(k)} \Psi_{0}^{-1}\left(\eta, \xi ; l=\mu_{k}\right)_{A B}
$$

$C^{(k)}$ is an arbitrary constant two-component vector, which together with $C_{0}^{(k)}$, provides all the moduli parameters of the general solitonic excitation of $g_{0}$. The other two-component vectors $N^{(k)}$ have more complicated forms and cannot be explicitly written with the same ease. $N^{(k)}$ are the solution vectors of the $n$th order linear system of algebraic equations

$$
\sum_{l=1}^{n} \Gamma_{k l} N_{A}^{(l)}=\frac{1}{\mu_{k}} \sum_{B} M_{B}^{(k)}\left(g_{0}\right)_{A B}
$$

where the $n \times n$ matrix $\Gamma$ was determined by Belinski and Sakharov

$$
\Gamma_{k l}=\frac{1}{\mu_{k} \mu_{l}-\alpha^{2}} \sum_{A, B} M_{A}^{(k)}\left(g_{0}\right)_{A B} M_{B}^{(l)} .
$$

Therefore, putting it all together we arrive at a concrete expression for the $n$-soliton excitation of $g_{0}$ : namely,

$$
g(\eta, \xi)=\left(1-\sum_{k=1}^{n} \frac{R_{k}}{\mu_{k}}\right) g_{0}
$$

A final issue is the overall normalization of the dressing matrix $\chi$. Using this last equation we find $\operatorname{det} g=\alpha^{2 n+2} \mu_{1}^{-2} \mu_{2}^{-2} \cdots \mu_{n}^{-2} \quad$ which differs from $\operatorname{det} g_{0}=\alpha^{2}$. Agreement is achieved by scaling $\chi$ with $\mu_{1} \mu_{2} \cdots \mu_{n} / \alpha^{n}$, and this is what we will assume from now on. The properly normalized $n$-soliton dressing matrices of $g_{0}$,

$$
\chi(l=0)=\frac{\mu_{1} \mu_{2} \cdots \mu_{n}}{\alpha^{n}}\left(1-\sum_{k=1}^{n} \frac{R_{k}}{\mu_{k}}\right),
$$

define specific group elements of the Geroch group. The normalization (2.25) is introduced to achieve consistency with the standard formulation of Geroch transformations that preserve detg.

Summarizing, if we apply this proceduce to any given string background $g_{0}, \lambda_{0}$ (more precisely, $\alpha \lambda_{0}$ to be in exact analogy for both sectors), we will obtain a generic $(n, m)$ solitonic excitation with $3 n+3 m$ continuous moduli. One of the difficulties to implement this construction in practice, apart from the problem of inverting the corresponding matrices $\Gamma$ for large $n$ and $m$, is to find the explicit solution $\Psi_{0}$ of
Eq. (2.10) (and its $\lambda$ counterpart) when an arbitrary background is used as seed. For this reason we will start from very simple seed solutions, knowing $\Psi_{0}$, and use the soliton technique to construct (and hence reinterpret in this context) the more complicated solutions that exist in the literature.

The Geroch group of the metric sector is the loop group $\hat{S} \mathrm{~L}(2)$, and when both sectors are taken into account the Geroch group becomes $\hat{\mathrm{S}} \mathrm{L}(2) \times \hat{\mathrm{S}} \mathrm{L}(2)=\hat{\mathrm{O}}(2,2)$. It is known in this case how to obtain the entire algebra by successive intertwining of continuous $T$ and $S$ transformations [4]. We briefly mention here that $4 \mathrm{D}$ backgrounds with two commuting isometries exhibit the obvious $\mathrm{O}(2,2)$ group of transformations on the space of solutions. These transformations are nonlocally realized in the axion-dilaton formulation of the theory and their generators are embedded in the algebra of the string Geroch group $\hat{O}(2,2)$ as follows: we use the zeromode subalgebra of the $g \hat{\mathrm{S} L}(2)$, say $T_{+}^{0}, T_{-}^{0}, T_{0}^{0}$, and the nonlocally realized SL(2) subalgebra of the $\lambda \hat{S} \mathrm{~L}(2)$, say $\widetilde{T}_{+}^{-1}, \widetilde{T}_{0}^{0}, \widetilde{T}_{-}^{1}$, that includes the \pm 1 modes. The continuous analogue of the $S$-duality SL(2) transformations are locally realized in the axion-dilaton formulation and correspond to the zero-mode generators $\widetilde{T}_{+}^{0}, \widetilde{T}_{-}^{0}, \widetilde{T}_{0}^{0}$, of the $\lambda \hat{S} \mathrm{~L}(2)$. Hence, by intertwining $\mathrm{O}(2,2)$ with $S$ we can generate after an infinite number of steps the entire $\hat{S} L(2)$ algebra of the axion-dilaton sector. To generate the other $\hat{S} \mathrm{~L}(2)$ we interchange the field variables $g \leftrightarrow \alpha \lambda$ and perform the same intertwining procedure. The exchange of the two sectors is a legitimate operation in this case because both $\sigma$ models have Euclidean signature, and this is also a $Z_{2}$ symmetry of the 2D reduced string background equations, leaving $f$ unaffected.

The $n$-soliton matrices (2.25) could also be described in terms of specific elements of the infinite-dimensional group of Geroch transformations obtained by other approaches [21]. We will not attempt here to decompose them in terms of more fundamental operations associated with successive intertwining of $T$ and $S$ transformations, but we note as an important property their commutativity in the following sense: an $\left(n+n^{\prime}\right)$ soliton can either be constructed directly from a seed background or it can be viewed as an $n^{\prime}$-solitonic excitation of the $n$-soliton, and similarly for $n \leftrightarrow n^{\prime}$. Since there is a systematic understanding of the group elements of soliton dressing, we think that is worth exploring further the precise meaning and the consequences of $U$ duality in this particular sector of string theory. Of course, much work remains to be done in this direction.

\section{III. $(1,1)$ SOLITONS AND CFT BACKGROUNDS}

According to the general framework of the previous section we may compute the simplest one-soliton solution of the Ernst $\sigma$ model, say Eq. (2.5), using as the seed metric

$$
g_{0}=\left(\begin{array}{cc}
\alpha^{2 s_{1}} & 0 \\
0 & \alpha^{2 s_{2}}
\end{array}\right), \quad s_{1}+s_{2}=1
$$

In a purely gravitational context this choice of the seed metric corresponds to a Kasner cosmological background. There are two special cases in this family, namely, 


$$
s_{1}=0, \quad s_{2}=1 ; \quad s_{1}=s_{2}=\frac{1}{2}
$$

which correspond to flat space (in polar coordinates) and an isotropic universe, respectively. Using Eq. (2.10) we may determine $\Psi_{0}$ for this background,

$$
\Psi_{0}(l)=\left(\begin{array}{ll}
\left(l^{2}+2 \beta l+\alpha^{2}\right)^{s_{1}} & 0 \\
0 & \left(l^{2}+2 \beta l+\alpha^{2}\right)^{s_{2}}
\end{array}\right) .
$$

We see clearly that $\Psi_{0}(l=0)=g_{0}$ as required on general grounds (2.13).

We will first derive the general form of the one-soliton solution, and then make various specializations according to the connections we would like to make later with 4D CFT backgrounds. The one-soliton background on $g_{0}$ (3.1) is obtained using only one pole located at

$$
\mu=\mu_{ \pm}=C_{0}-\beta \pm \sqrt{\left(C_{0}-\beta\right)^{2}-\alpha^{2}} .
$$

This pole is positioned on the real axis of the complex $l$ plane provided that

$$
\left(C_{0}-\beta\right)^{2} \geqslant \alpha^{2} \text {. }
$$

Otherwise, we will be forced to consider more complicated double-soliton solutions, since complex poles always come in pairs. Then, the physical one-soliton matrix (after normalization with $\mu / \alpha$ ) reads

$$
g_{1}=\frac{\alpha}{\mu A}\left(\begin{array}{ll}
C_{2}^{2}\left(2 C_{0} \mu\right)^{2 s_{1}} \mu^{2}+C_{1}^{2}\left(2 C_{0} \mu\right)^{2 s_{2}} \alpha^{4 s_{1}} & 2 C_{0} C_{1} C_{2} \mu\left(\alpha^{2}-\mu^{2}\right) \\
2 C_{0} C_{1} C_{2} \mu\left(\alpha^{2}-\mu^{2}\right) & C_{1}^{2}\left(2 C_{0} \mu\right)^{2 s_{2}} \mu^{2}+C_{2}^{2}\left(2 C_{0} \mu\right)^{2 s_{1}} \alpha^{4 s_{2}}
\end{array}\right),
$$

where

$$
A=C_{1}^{2}\left(2 C_{0} \mu\right)^{2 s_{2}} \alpha^{2 s_{1}}+C_{2}^{2}\left(2 C_{0} \mu\right)^{2 s_{1}} \alpha^{2 s_{2}} .
$$

If we were to apply the same construction to the axiondilaton Ernst $\sigma$ model (2.6), we should have scaled $\alpha$ in front of the seed matrix (3.1), since $g_{0}$ behaves the same way as $\sqrt{\operatorname{det} g} \lambda_{0}=\alpha \lambda_{0}$. Let us begin with a background having

$$
e^{-2 \Phi_{0}}=\alpha^{2 s_{2}^{\prime}-1}, \quad b_{0}=0
$$

with $s_{1}^{\prime}+s_{2}^{\prime}=1$ as well. Scaling out $\alpha$ from the general form of the one-soliton solution, we find that the new axiondilaton system is given by the configuration

$$
\begin{gathered}
e^{-2 \Phi_{1}}=\mu \frac{C_{1}^{\prime 2}\left(2 C_{0}^{\prime} \mu^{\prime}\right)^{2 s_{2}^{\prime}} \alpha^{2 s_{1}^{\prime}}+C_{2}^{\prime 2}\left(2 C_{0}^{\prime} \mu^{\prime}\right)^{2 s_{1}^{\prime}} \alpha^{2 s_{2}^{\prime}}}{C_{1}^{\prime 2}\left(2 C_{0}^{\prime} \mu^{\prime}\right)^{2 s_{2}^{\prime}} \alpha^{4 s_{1}^{\prime}}+C_{2}^{\prime 2}\left(2 C_{0}^{\prime} \mu^{\prime}\right)^{2 s_{1}^{\prime}} \mu^{\prime 2}}, \\
b_{1}=\frac{2 C_{0}^{\prime} C_{1}^{\prime} C_{2}^{\prime} \mu^{\prime}\left(\alpha^{2}-\mu^{\prime 2}\right)}{C_{1}^{\prime 2}\left(2 C_{0}^{\prime} \mu^{\prime}\right)^{2 s_{2}^{\prime}} \alpha^{4 s_{1}^{\prime}}+C_{2}^{\prime 2}\left(2 C_{0}^{\prime} \mu^{\prime}\right)^{2 s_{1}^{\prime}} \mu^{\prime 2}},
\end{gathered}
$$

where the primes are used to distinguish the parameters of the axion-dilaton system from those of the metric moduli.

Suppose now we are combining both sectors to construct the $(1,1)$ soliton starting from the following solution of the string background equations in the Einstein frame:

$$
\begin{gathered}
d s^{2}=-d \alpha^{2}+d \beta^{2}+\alpha\left(d z^{2}+d w^{2}\right), \\
b_{0}=0, \quad e^{-2 \Phi_{0}}=\alpha .
\end{gathered}
$$

This particular choice of the seed background is very special in that it is $T$ dual to $F^{(4)}$, i.e., the flat space metric with zero dilaton and antisymmetric tensor fields. To see this we translate Eq. (3.11) in the $\sigma$-model frame and perform $T$ duality with respect to the Killing coordinate $\beta$, which yields the purely gravitational background

$$
d s^{2}=-\frac{1}{\alpha} d \alpha^{2}+\alpha d \beta^{2}+d z^{2}+d w^{2}
$$

Introducing coordinates

$$
x=2 \sqrt{\alpha} \cosh \frac{\beta}{2}, \quad y=2 \sqrt{\alpha} \sinh \frac{\beta}{2},
$$

the metric (3.13) assumes the flat space form $d s^{2}=-d x^{2}+d y^{2}+d z^{2}+d w^{2}$. Actually, it is immediately recognized that (3.14) is a Rindler transformation of the $2 \mathrm{D}$ Minkowski space $(x, y)$ with $(\ln \alpha, \beta)$ providing the corresponding pair of Rindler coordinates. Hence, we start from the 2D Rindler wedge times a flat 2D Euclidean space parametrized by the other two coordinates $(z, w)$, and use its $T$-dual face as seed string background.

We notice that our Ansatze (3.11) and (3.12) for the seed background imply the following choice of the Kasner-type parameters for the two sectors:

$$
\begin{gathered}
g: \quad s_{1}=s_{2}=\frac{1}{2}, \\
\lambda: \quad s_{1}^{\prime}=0, \quad s_{2}^{\prime}=1 .
\end{gathered}
$$

Then, the resulting $(1,1)$ soliton simplifies considerably and in the Einstein frame is given by

$$
g_{1}=\frac{1}{C_{1}^{2}+C_{2}^{2}}\left(\begin{array}{cc}
C_{1}^{2} \frac{\alpha^{2}}{\mu}+C_{2}^{2} \mu & C_{1} C_{2}\left(\frac{\alpha^{2}}{\mu}-\mu\right) \\
C_{1} C_{2}\left(\frac{\alpha^{2}}{\mu}-\mu\right) & C_{1}^{2} \mu+C_{2}^{2} \frac{\alpha^{2}}{\mu}
\end{array}\right),
$$




$$
\begin{gathered}
e^{-2 \Phi_{1}=} \frac{1}{4 C_{0}^{\prime 2} C_{1}^{\prime 2}+C_{2}^{\prime 2}}\left(4 C_{0}^{\prime 2} C_{1}^{\prime 2} \mu^{\prime}+C_{2}^{\prime 2} \frac{\alpha^{2}}{\mu^{\prime}}\right) \\
b_{1}=\frac{2 C_{0}^{\prime} C_{1}^{\prime} C_{2}^{\prime}}{4 C_{0}^{\prime 2} C_{1}^{\prime 2}+C_{2}^{\prime 2}}\left(\frac{\alpha^{2}}{\mu^{\prime}}-\mu^{\prime}\right)
\end{gathered}
$$

As for the conformal factor $f$, which follows by integration of Eqs. (2.8) and (2.9), we find after some lengthy computation the result

$$
f_{1}=\frac{\mu\left(4 C_{0}^{\prime 2} C_{1}^{\prime 2} \mu^{\prime 2}+C_{2}^{\prime 2} \alpha^{2}\right)}{\left(\alpha^{2}-\mu^{2}\right)\left(\alpha^{2}-\mu^{\prime 2}\right)}
$$

up to an overall numerical factor, whereas $f_{0}=1$ by inspecting Eq. (3.11).

There is an ambiguity to choose $\mu_{+}$or $\mu_{-}$in Eqs. (3.17)-(3.19), but since

$$
\mu_{ \pm}=\frac{\alpha^{2}}{\mu_{\mp}}
$$

the two choices yield the same result provided that in the metric soliton moduli space $\left(C_{1}, C_{2}\right) \rightarrow\left(-C_{2}, C_{1}\right)$. Similarly, in the axion-dilaton sector the two choices $\mu_{ \pm}^{\prime}$ are equivalent provided that $\left(C_{1}^{\prime}, C_{2}^{\prime}\right) \rightarrow\left(-C_{2}^{\prime} / 2 C_{0}^{\prime}, 2 C_{0}^{\prime} C_{1}^{\prime}\right)$. Hence, in the following we may choose, without loss of generality,

$$
\begin{gathered}
\mu=C_{0}-\beta+\sqrt{\left(C_{0}-\beta\right)^{2}-\alpha^{2}}, \\
\mu^{\prime}=C_{0}^{\prime}-\beta+\sqrt{\left(C_{0}^{\prime}-\beta\right)^{2}-\alpha^{2}} .
\end{gathered}
$$

Next, we show how to obtain the string backgrounds associated with the two coset models $\mathrm{SL}(2) \times \mathrm{SU}(2) /$ $\mathrm{SO}(1,1) \times \mathrm{U}(1)$ and $\mathrm{SL}(2) / \mathrm{SO}(1,1) \times \mathrm{SO}(1,1)^{2}$ by making appropriate choices of the moduli parameters in the $6 \mathrm{D}$ space of solutions we have obtained.

(i) Nappi-Witten universe: In the $g$ sector of the general $(1,1)$ soliton solution we choose

$$
C_{1}=0
$$

which gives rise to a diagonal metric with components

$$
\begin{aligned}
& g_{z z}=C_{0}-\beta+\sqrt{\left(C_{0}-\beta\right)^{2}-\alpha^{2}}, \\
& g_{w w}=C_{0}-\beta-\sqrt{\left(C_{0}-\beta\right)^{2}-\alpha^{2}}
\end{aligned}
$$

independent of $C_{2}$. For the axion-dilaton sector we set

$$
C_{0}^{\prime}=1, \quad \frac{C_{2}^{\prime}}{2 C_{1}^{\prime}}=\frac{\sin \theta-1}{\cos \theta},
$$

where $\theta$ is an arbitrary numerical constant. Hence, choosing $\mu_{+}^{\prime}$ we also fix

$$
\begin{gathered}
e^{-2 \Phi}=1-\beta+\sin \theta \sqrt{(1-\beta)^{2}-\alpha^{2}}, \\
b=\cos \theta \sqrt{(1-\beta)^{2}-\alpha^{2}},
\end{gathered}
$$

while the conformal factor in the Einstein frame is determined according to Eq. (3.20).

We claim that this solution corresponds to the cosmological background found by Nappi and Witten while considering the $\mathrm{SL}(2) \times \mathrm{SU}(2) / \mathrm{SO}(1,1) \times \mathrm{U}(1) \mathrm{CFT}$ coset. In this regard, the numerical parameter $\theta$ that was introduced in Eq. (3.25) will be shown to describe the arbitrariness in the gauging of this coset. For this purpose we also choose

$$
C_{0}=-1 \text {, }
$$

thus describing the same $\theta$-dependent string background for any point in the soliton moduli space that is restricted by Eqs. (3.23), (3.25), and (3.28).

The construction is rather formal up to now, while making various seemingly unjustified choices of the free parameters. At this point we introduce coordinates $X^{0}, X^{1}$ in terms of $\alpha, \beta$ given by

$$
\alpha=\sin 2 X^{0} \sin 2 X^{1}, \quad \beta=\cos 2 X^{0} \cos 2 X^{1},
$$

thus also restricting the range of $\alpha$ and $\beta$ as $X^{0}$ and $X^{1}$ range from 0 to $\pi / 2$. This is a good choice because

$$
e^{-2 \Phi}=1-\cos 2 X^{0} \cos 2 X^{1}+\sin \theta\left(\cos 2 X^{0}-\cos 2 X^{1}\right)
$$

is manifestly real and positive, as should be expected for an honest dilaton field. For the axion we find

$$
b=\cos \theta\left(\cos 2 X^{0}-\cos 2 X^{1}\right) .
$$

At first sight it seems that these choices are not good for the metric sector (3.24), since

$$
g_{z z}=-4 \sin ^{2} X^{0} \sin ^{2} X^{1}, \quad g_{w w}=-4 \cos ^{2} X^{0} \cos ^{2} X^{1},
$$

and the signature turns out to be -- instead of ++ . Recall, however, the way we have obtained the physical metric in the soliton construction of Sec. II. There, we had to scale $\chi(l=0)$ accordingly so that $\operatorname{det} g=\operatorname{det} g_{0}=\alpha^{2}$. The scaling was $\mu / \alpha$ for the one-soliton, but equally well we could have taken $-\mu / \alpha$. The latter choice renders the signature of $g$ physical, i.e., ++ , and there is no contradiction.

To make exact contact with the Nappi-Witten cosmological background we introduce coordinates $X^{2}$ and $X^{3}$ by scaling

$$
w=\sqrt{\frac{1+\sin \theta}{2}} X^{2}, \quad z=\sqrt{\frac{1-\sin \theta}{2}} X^{3}
$$

and compute the full metric in the $\sigma$-model frame. The final result reads

$$
\begin{aligned}
d s_{(\sigma)}^{2}= & -\left(d X^{0}\right)^{2}+\left(d X^{1}\right)^{2} \\
& +\frac{2}{1-\cos 2 X^{0} \cos 2 X^{1}+\sin \theta\left(\cos 2 X^{0}-\cos 2 X^{1}\right)} \\
& \times\left[(1+\sin \theta) \cos ^{2} X^{0} \cos ^{2} X^{1}\left(d X^{2}\right)^{2}\right. \\
& \left.+(1-\sin \theta) \sin ^{2} X^{0} \sin ^{2} X^{1}\left(d X^{3}\right)^{2}\right] .
\end{aligned}
$$


We also compute the antisymmetric tensor field from the axion and find that all its components are zero apart from

$$
B_{23}=\frac{1}{2} \frac{\cos 2 X^{0}-\cos 2 X^{1}+\sin \theta\left(1-\cos 2 X^{0} \cos 2 X^{1}\right)}{1-\cos 2 X^{0} \cos 2 X^{1}+\sin \theta\left(\cos 2 X^{0}-\cos 2 X^{1}\right)} .
$$

This is precisely the result that was obtained in the semiclassical limit of the $\mathrm{SL}(2) \times \mathrm{SU}(2) / \mathrm{SO}(1,1) \times \mathrm{U}(1)$ coset model having an arbitrary parameter $\theta$ that specifies the gauging [12].

So, according to this, the Nappi-Witten universe can be created from flat space starting from (a suitably restricted part of) the Rindler wedge, performing a $T$ duality transformation and then a $(1,1)$ soliton dressing. Consequently, our procedure completely determines the group element of the string Geroch group $\hat{O}(2,2)$ that connects classically the two backgrounds. The Nappi-Witten background describes a closed expanding and recontracting universe as $X^{0}$ varies from 0 (big bang) to $\pi / 2$ (big crunch). These two authors performed an in-depth analysis of the model noting that for

$$
\frac{1-\sin \theta}{\cos \theta}=\text { rational number }
$$

$X^{0}=0$ or $\pi / 2$ are orbifold singularities. Also, away from the special values $X^{1}=0$ or $\pi / 2$, respectively, these are singularities in the causal structure of spacetime rather than curvature singularities.

This cosmological solution is positioned in the entire moduli space of $(1,1)$ solitons as follows: consider the 3D subspace with axis labeled by $C_{2}, C_{1}^{\prime}$, and $C_{2}^{\prime}$, while keeping the other coordinates fixed to their chosen values $C_{1}=0, C_{0}^{\prime}=1=-C_{0}$; if we draw all $2 \mathrm{D}$ planes having the $C_{2}$ line as common axis, then every point on each such plane will correspond to the same solution, while rotating planes change $\theta$. In this description the criterion (3.36) for having orbifold singularities is equivalent to considering rational values for the slope of the solution plane, which is given by $C_{2}^{\prime} / C_{1}^{\prime}$ according to Eq. (3.25).

Concluding we mention that the points of the moduli space with the same restrictions as before, but with $C_{2}^{\prime}=0$, yield (a suitable analytic continuation of) the background $\mathrm{SL}(2) / \mathrm{SO}(1,1) \times \mathrm{SU}(2) / \mathrm{U}(1)$. Using the parametrization (3.29) it follows from our general expression that the axion field is zero, the dilaton field is

$$
e^{-2 \Phi}=\cos ^{2} X^{0} \sin ^{2} X^{1},
$$

and the metric in the $\sigma$-model frame is diagonal,

$$
d s_{(\sigma)}^{2}=-\left(d X^{0}\right)^{2}+\left(d X^{1}\right)^{2}+\cot ^{2} X^{1}\left(d X^{2}\right)^{2}+\tan ^{2} X^{0}\left(d X^{3}\right)^{2} .
$$

It describes a suitable real form of the direct product of two 2D black hole cosets. This background was used in [14] to obtain the complete Nappi-Witten solution by $\mathrm{O}(2,2)$ transformations. If $(1-\sin \theta) / \cos \theta$ takes only integer values, the transformation is in $\mathrm{O}(2,2 ; Z)$ and the underlying backgrounds are equivalent as exact conformal field theories. It is interesting to note that the transformation that provides the $(1,1)$ soliton dressing already contains in it the correspond- ing $\mathrm{O}(2,2)$ group elements; but it also contains much more that allow for a flat space derivation of these CFT backgrounds.

(ii) The coset $\mathrm{SL}(2) / \mathrm{SO}(1,1) \times \mathrm{SO}(1,1)^{2}$ : Following the same construction as above we will now specify other points in the moduli space of soliton solutions (3.17)-(3.19) that lead to the semiclassical geometry of the $\operatorname{SL}(2) / \operatorname{SO}(1,1)$ $\times \mathrm{SO}(1,1)^{2}$ coset.

We choose $C_{1}=0$ for the metric sector, thus arriving at the same expression (3.24) as before, while for the axiondilaton sector we let $C_{2}^{\prime}=0$. In this case we find

$$
\begin{gathered}
e^{-2 \Phi}=C_{0}^{\prime}-\beta+\sqrt{\left(C_{0}^{\prime}-\beta\right)^{2}-\alpha^{2}}, \\
b=0 .
\end{gathered}
$$

We furthermore let

$$
C_{0}=C_{0}^{\prime},
$$

and introduce the coordinate transformation

$$
\alpha=\frac{1}{2} e^{2 X^{1}} \sinh 2 X^{0}, \quad \beta=C_{0}-\frac{1}{2} e^{2 X^{1}} \cosh 2 X^{0},
$$

which clearly has $\left(C_{0}-\beta\right)^{2} \geqslant \alpha^{2}$ as required for reality. We find in this parametrization

$$
e^{-2 \Phi}=e^{2 X^{1}} \cosh ^{2} X^{0},
$$

while the antisymmetric tensor field is zero and the metric is diagonal. In the $\sigma$-model frame, also setting $w=X^{2}$ and $z=X^{3}$, the metric assumes the form

$$
d s_{(\sigma)}^{2}=-\left(d X^{0}\right)^{2}+\left(d X^{1}\right)^{2}+\tanh ^{2} X^{0}\left(d X^{2}\right)^{2}+\left(d X^{3}\right)^{2},
$$

and the resulting background coincides with the geometry of the coset $\mathrm{SL}(2) / \mathrm{SO}(1,1) \times \mathrm{SO}(1,1)^{2}$ as it was advertised.

The wormhole background will be discussed separately in Sec. V using 2D solitons in Euclidean space.

\section{BLACK HOLES AS 2D (2,0) SOLITONS}

In this section we briefly review for completeness the interpretation of ordinary 4D black holes as $2 \mathrm{D}(2,0)$ solitons, filling up some of the intermediate steps of the calculation [19] as well. We use as a starting point the flat space metric in polar coordinates,

$$
d s^{2}=d \alpha^{2}+d \beta^{2}+\alpha^{2} d \varphi^{2}-d \tau^{2},
$$

for which the matrix $\Psi$ of the linearized system (2.10) for stationary axisymmetric metrics is

$$
\Psi_{0}(l)=\left(\begin{array}{cc}
\alpha^{2}-2 \beta l-l^{2} & 0 \\
0 & -1
\end{array}\right) .
$$

We point out a few differences between this case and the cosmological setting of the two previous sections. Here, the 2D space $(\alpha, \beta)$ has Euclidean signature, while the $\sigma$-model $g$ is Lorentzian. As a result, one has to take into account various sign changes in order to adopt the general 
soliton construction to stationary axisymmetric metrics; in particular, Eq. (2.18) changes to

$$
\mu_{k}^{2}+2\left(\beta-C_{0}^{(k)}\right) \mu_{k}-\alpha^{2}=0,
$$

and the factor $\mu_{k} \mu_{l}-\alpha^{2}$ in Eq. (2.23) changes to $\mu_{k} \mu_{l}+\alpha^{2}$. Analogous changes have to be introduced in the linearized system of equations $(2.10)-(2.12)$ and the differential equations (2.19) for the poles $\mu_{k}$. Also, the $n$-soliton transformation of a seed background $g_{0}$ yields $\operatorname{det} g=(-1)^{n} \alpha^{2 n+2} \mu_{1}^{-2} \mu_{2}^{-2} \cdots \mu_{n}^{-2}$ and the normalization (2.25) is the same as before for $n$ even. If $n$ is odd, however, the signature of the soliton metric $g$ changes sign to ++ , which is is not acceptable. For this reason the simplest physical soliton to construct is the double-soliton solution on flat space (4.1).

The present version of the formalism will also become relevant in the next section, while considering Euclidean gravitational solutions of the string background equations. In that case the axion-dilaton system corresponds to a Lorentzian $\sigma$-model $\lambda$, and the explicit construction of its solitons will require the modifications we are considering here.

After some calculation we find that the general $(2,0)$ soliton on the purely gravitational background (4.1) has metric components

$$
g_{\tau \varphi}=2\left(\mu_{1}-\mu_{2}\right)\left(\alpha^{2}+\mu_{1} \mu_{2}\right) \frac{N_{1}}{D}, \quad g_{\tau \tau}=-4 \mu_{1} \mu_{2} \frac{N_{2}}{D},
$$

where

$$
\begin{gathered}
N_{1}=A \Sigma\left(\alpha^{2}+\mu_{1}^{2}\right)\left(\alpha^{2}+\mu_{2}^{2}\right)+A M \alpha^{2}\left(\mu_{1}^{2}-\mu_{2}^{2}\right) \\
-B \Sigma\left(\alpha^{4}-\mu_{1}^{2} \mu_{2}^{2}\right), \\
N_{2}=\Sigma^{2}\left(\alpha^{2}+\mu_{1} \mu_{2}\right)^{2}-A^{2} \alpha^{2}\left(\mu_{1}-\mu_{2}\right)^{2}, \\
D=\left[(\Sigma+M) \mu_{1}+(\Sigma-M) \mu_{2}\right]^{2}\left(\alpha^{2}+\mu_{1} \mu_{2}\right)^{2}+\left[(A-B) \alpha^{2}\right. \\
\left.-(A+B) \mu_{1} \mu_{2}\right]^{2}\left(\mu_{1}-\mu_{2}\right)^{2},
\end{gathered}
$$

while $g_{\varphi \varphi}$ is determined by the condition $\operatorname{det} g=-\alpha^{2}$. Also, the corresponding conformal factor turns out to be

$$
f=\frac{\mu_{1} \mu_{2}}{4\left(\mu_{1}-\mu_{2}\right)^{2}\left(\alpha^{2}+\mu_{1}^{2}\right)\left(\alpha^{2}+\mu_{2}^{2}\right)\left(\alpha^{2}+\mu_{1} \mu_{2}\right)^{2}} D,
$$

whereas $f_{0}=1$. In the above expressions the parameters $A$, $B, M$, and $\Sigma$ are the special combinations of the $C^{(k)}$ moduli,

$$
\begin{gathered}
C_{1}^{(1)} C_{2}^{(2)}=(\Sigma-M) C_{0}^{(1)}, \quad C_{1}^{(1)} C_{1}^{(2)}=2(A-B) C_{0}^{(1)} C_{0}^{(2)} \\
C_{2}^{(1)} C_{1}^{(2)}=-(\Sigma+M) C_{0}^{(2)} \\
C_{2}^{(1)} C_{2}^{(2)}=\frac{1}{2}(A+B)
\end{gathered}
$$

which clearly satisfy the condition

$$
\Sigma^{2}=M^{2}-A^{2}+B^{2}
$$

Also, using an appropriate shift of $\beta$ we may fix,

$$
\Sigma=\frac{1}{2}\left(C_{0}^{(1)}-C_{0}^{(2)}\right), \quad Z=\frac{1}{2}\left(C_{0}^{(1)}+C_{0}^{(2)}\right) .
$$

We introduce now the change of variables

$$
\alpha=\sqrt{(r-M)^{2}-\Sigma^{2}} \sin \theta, \quad \beta-Z=(r-M) \cos \theta,
$$

and substitute for $\mu_{1}$ and $\mu_{2}$. Provided that we choose the solutions $\mu_{k+}$ of Eq. (4.3), we obtain

$$
\mu_{1}=2(r-M+\Sigma) \sin ^{2} \frac{\theta}{2}, \quad \mu_{2}=2(r-M-\Sigma) \sin ^{2} \frac{\theta}{2} .
$$

Hence, the two-soliton solution depends only on three moduli $A, B$, and $M$, while $\Sigma$ is fixed by Eq. (4.10) and $Z$ does not appear anywhere. It is also useful to introduce the change of variable

$$
t=-\tau+2 A \varphi
$$

and identify $t$ with the time coordinate. Then, substituting in Eq. (4.4)-(4.8) we may compute the explicit form of the two-soliton metric in the coordinates $(r, \theta, \varphi, t)$.

The special case $A=B=0$ is the simplest, since the resulting two-soliton metric is diagonal,

$$
d s^{2}=r^{2}\left(d \theta^{2}+\sin ^{2} \theta d \varphi^{2}\right)+\frac{r}{r-2 M} d r^{2}-\frac{r-2 M}{r} d t^{2},
$$

and coincides with the Schwarzschild metric. In the more general situation we obtain the Kerr metric with mass parameter $M$, rotation parameter $A$, and NUT parameter $B$ that describes the behavior of the 4D metric at infinity. The result of the two-soliton construction precisely yields the complete Kerr metric in Boyer-Lindquist coordinates,

$$
\begin{aligned}
d s^{2}= & \frac{1}{r^{2}+(B-A \cos \theta)^{2}}\left\{-\left[(r-M)^{2}-\Sigma^{2}-A^{2} \sin ^{2} \theta\right] d t^{2}\right. \\
& +\left[\sin ^{2} \theta\left(r^{2}+A^{2}+B^{2}\right)^{2}-\left((r-M)^{2}-\Sigma^{2}\right)(2 B \cos \theta\right. \\
& \left.\left.+A \sin ^{2} \theta\right)^{2}\right] d \varphi^{2}+4\left[B \cos \theta\left((r-M)^{2}-\Sigma^{2}\right)\right. \\
& \left.\left.-A \sin ^{2} \theta\left(M r+B^{2}\right)\right] d t d \varphi\right\}+\left[r^{2}+(B-A \cos \theta)^{2}\right] \\
& \times\left(d \theta^{2}+\frac{1}{(r-M)^{2}-\Sigma^{2}} d r^{2}\right)
\end{aligned}
$$

We note finally that the Lorentzian analogue of the selfdual Taub-NUT metric corresponds to the limits $\Sigma=0=A$ and $M= \pm i B$. These limits, however, are somewhat peculiar in the two-soliton sector because all three quantities $N_{1}$, $N_{2}, D$ become zero, and the two real poles (4.13) coincide.

\section{EUCLIDEAN (0,2) SOLITONS}

We describe now how axionic instanton solutions of the Euclidean string background equations can be accommodated into the present scheme. More specifically, we study the moduli space of $(0,2) 2 \mathrm{D}$ solitons and determine the 
specific choice of parameters that give rise to the wormhole (and other related backgrounds) as solitons on flat space. It is true that these backgrounds were originally introduced as soliton solutions of the 10D heterotic string theory (see, for instance, [14] and references therein), but their description as $2 \mathrm{D}$ solitons of the reduced $\beta$-function equations using inverse-scattering methods appears to be new.

We consider the general form of the $(0,2)$ soliton solutions starting from 4D flat Euclidean space. It will be convenient for later use to consider as seed metric

$$
\begin{aligned}
d s^{2}= & \frac{1}{2 \sqrt{\alpha^{2}+\beta^{2}}}\left(d \alpha^{2}+d \beta^{2}\right)+\left(\sqrt{\alpha^{2}+\beta^{2}}-\beta\right) d \psi^{2} \\
& +\left(\sqrt{\alpha^{2}+\beta^{2}}+\beta\right) d \tau^{2}
\end{aligned}
$$

with $\operatorname{det} g=\alpha^{2}$, instead of the Euclidean version of Eq. (4.1). However, the explicit form of the metric background will be used only after completing the soliton construction in the axion-dilaton sector. The seed $\Phi$ and $b$ are zero, and the metric (5.1) is flat as can be seen using the transformation

$$
\alpha=\frac{1}{2} e^{2 \rho} \sin 2 \varphi, \quad \beta=\frac{1}{2} e^{2 \rho} \cos 2 \varphi,
$$

or introducing $z=\exp (\rho+i \tau) \cos \varphi, w=\exp (\rho+i \psi) \sin \varphi$ that yields $d s^{2}=d z d \bar{z}+d w d \bar{w}$.

The axion-dilaton sector has Lorentzian signature [we should replace $b^{2}+e^{-4 \Phi}$ with $b^{2}-e^{-4 \Phi}$ in Eq. (2.1), since the relevant conjugate pair of field variables is now $S_{ \pm}=b \pm e^{-2 \Phi}$ instead of $\left.b \pm i e^{-2 \Phi}\right]$, and therefore, the $(0,2)$ soliton calculation is analogous to $(2,0)$ metric solitons of stationary axisymmetric gravity. There is a difference with the analysis of the previous section, however, in that our normalized axion-dilaton seed matrix $\alpha \lambda_{0}=\operatorname{diag}(\alpha,-\alpha)$ is "isotropic," while $g_{0}=\operatorname{diag}\left(\alpha^{2},-1\right)$ in Eq. (4.1). It is, therefore, appropriate in the present case to consider

$$
\Psi_{0}(l)=\sqrt{\alpha^{2}-2 \beta l-l^{2}}\left(\begin{array}{cc}
1 & 0 \\
0 & -1
\end{array}\right) .
$$

Explicit calculation shows that the $(0,2)$ axion-dilaton soliton fields are

$$
e^{-2 \Phi}=2 \mu_{1} \mu_{2} \frac{N_{1}^{\prime}}{D^{\prime}}, \quad b=\left(\mu_{1}-\mu_{2}\right)\left(\alpha^{2}+\mu_{1} \mu_{2}\right) \frac{N_{2}^{\prime}}{D^{\prime}},
$$

where

$$
\begin{gathered}
N_{1}^{\prime}=B^{2} \alpha^{2}\left(\mu_{1}-\mu_{2}\right)^{2}+C^{2}\left(\alpha^{2}+\mu_{1} \mu_{2}\right)^{2}, \\
N_{2}^{\prime}=A C\left(\mu_{1}+\mu_{2}\right)\left(\alpha^{2}+\mu_{1} \mu_{2}\right) \\
+B D\left(\mu_{1}-\mu_{2}\right)\left(\alpha^{2}-\mu_{1} \mu_{2}\right), \\
D^{\prime}=C^{2}\left(\mu_{1}^{2}+\mu_{2}^{2}\right)\left(\alpha^{2}+\mu_{1} \mu_{2}\right)^{2}-B^{2}\left(\mu_{1}-\mu_{2}\right)^{2}\left(\alpha^{4}+\mu_{1}^{2} \mu_{2}^{2}\right) \\
-\left(\mu_{1}-\mu_{2}\right)\left(\alpha^{2}+\mu_{1} \mu_{2}\right)\left[A B\left(\mu_{1}-\mu_{2}\right)\left(\alpha^{2}-\mu_{1} \mu_{2}\right)\right. \\
\left.+C D\left(\mu_{1}+\mu_{2}\right)\left(\alpha^{2}+\mu_{1} \mu_{2}\right)\right] .
\end{gathered}
$$

Here, we have used for convenience the notation

$$
\begin{gathered}
C_{1}^{(1)} C_{1}^{(2)}=\frac{1}{2}(A+B), \quad C_{1}^{(1)} C_{2}^{(2)}=-\frac{1}{2}(C+D), \\
C_{2}^{(1)} C_{2}^{(2)}=\frac{1}{2}(A-B), \quad C_{1}^{(2)} C_{2}^{(1)}=\frac{1}{2}(C-D),
\end{gathered}
$$

which satisfy the relation

$$
C^{2}=D^{2}-A^{2}+B^{2} .
$$

So, the soliton moduli depends on four parameters in this case, $A, B, C$, and one of the $C_{0}^{(k)}$, since the other one can be absorbed by shifting $\beta$.

Searching for an axionic instanton solution in this moduli space we notice that if

$$
A= \pm D, \quad B^{2}=C^{2}
$$

we obtain

$$
e^{-2 \Phi} \mp b=1 .
$$

One can also prove relatively easily that there are no other axionic instanton solutions in this sector. Moreover, from all soliton constructions we have considered so far, only this example exhibits axionic instanton solutions.

Next, we determine the explicit form of the solution choosing $A=D$ and $B=C$ for concreteness. Substituting in Eq. (5.4) we obtain

$$
e^{2 \Phi}=\frac{D^{\prime}}{2 \mu_{1} \mu_{2} N_{1}^{\prime}}=1-\frac{A}{B} \frac{\left(\mu_{1}-\mu_{2}\right)\left(\alpha^{2}+\mu_{1} \mu_{2}\right)}{\mu_{2}\left(\alpha^{2}+\mu_{1}^{2}\right)} .
$$

Further manipulation using Eq. (4.3) yields

$$
e^{2 \Phi}=1-2 \frac{A}{B}\left(C_{0}^{(1)}-C_{0}^{(2)}\right) \frac{1}{\mu_{1}+\frac{\alpha^{2}}{\mu_{1}}}
$$

and so the dilaton depends only on $\mu_{1}$. Choosing the solution $\mu_{+}$and taking into account that $\mu_{-}=-\alpha^{2} / \mu_{+}$in this case, we finally arrive at the result

$$
e^{2 \Phi}=1+\frac{M}{\sqrt{\left(C_{0}^{(1)}-\beta\right)^{2}+\alpha^{2}}}, \quad M=\left(C_{0}^{(2)}-C_{0}^{(1)}\right) \frac{A}{B} .
$$

$C_{0}^{(1)}$ can be absorbed by shifting $\beta$, and hence for all purposes it may be set equal to zero.

We also note for completeness that for $A=D$, but $B=-C$, the dilaton depends only on $\mu_{2}$ and the result turns out to be essentially the same up to an interchange of $C_{0}^{(1)}$ and $C_{0}^{(2)}$. Similar remarks apply to the axionic anti-instanton case $A=-D$ with $B= \pm C$.

The solution we have obtained in this fashion can be put together with the flat space metric (5.1) to yield in the Einstein frame the $4 \mathrm{D}$ string background

$$
d s^{2}=e^{2 \rho}\left(d \rho^{2}+d \varphi^{2}+\sin ^{2} \varphi d \psi^{2}+\cos ^{2} \varphi d \tau^{2}\right),
$$




$$
e^{2 \Phi}=1+2 M e^{-2 \rho}, \quad b=e^{-2 \Phi}+\text { const. }
$$

We have used the change of coordinates (5.2) and the fact that for axionic instantons there is no contribution to the conformal factor $f$ coming from the $\lambda$ sector [see Eqs. (2.8) and (2.9)]. This configuration is actually known as the wormhole solution. A related version of it, which represents only the throat of the wormhole and coincides with the semiclassical geometry of the $\mathrm{SU}(2) \times \mathrm{U}(1)$ WZW model, consists of a dilaton field with different boundary condition in that the constant term in Eq. (5.16) is missing, but with the same Einstein metric. These two models are related to each other by an $\operatorname{SL}(2, R)$ transformation (the continuous counterpart of $S$ duality), which keeps the axionic instanton condition invariant, say $S_{-}$constant, and simply shifts $e^{2 \Phi}$ by a constant.

We finally point out that higher solitonic excitations of the axion-dilaton sector might be interesting to consider in this case. We have determined the solution of the linearized system for the $\lambda$ sector of the semiclassical background of the model $\mathrm{SU}(2) \times \mathrm{U}(1)$,

$$
\Psi_{\mathrm{wh}}(l)=\left(\begin{array}{ll}
\frac{\alpha^{2}-\beta l}{2 \sqrt{\left(\alpha^{2}+\beta^{2}\right)\left(\alpha^{2}-2 \beta l-l^{2}\right)}} & \sqrt{\alpha^{2}-2 \beta l-l^{2}} \\
\sqrt{\alpha^{2}-2 \beta l-l^{2}} & 0
\end{array}\right) .
$$

However, we are not in a position at this moment to give a good spacetime interpretation to its multi-soliton excitations. It might turn out an interesting geometrical problem and we hope to return to it elsewhere.

\section{CONCLUSIONS AND DISCUSSION}

In this paper we have considered solitonic solutions of the $2 \mathrm{D}$ reduced $\beta$-function equations for gravitational string backgrounds with axion and dilaton fields. We found that many known solutions that admit an exact conformal field theory description arise as simple solitonic excitations of flat space, or its $T$-dual face, depending on the particular example. These backgrounds include cosmological solutions, as well as 4D blackholes and wormholes. It should be also interesting to consider further generalizations to colliding gravitational plane wave solutions of string theory, thus extending the results of [20] to strings.

A key point in implementing our construction is the nontrivial interplay between the coordinate and the solutiongenerating (Geroch-type) transformations. These two kinds of transformations do not commute with each other, and we should always use a coordinate system in the seed background with nonconstant detg. The Geroch transformations leave detg invariant, and only after the solitonic dressing is performed we may transform the new background into any other suitable coordinate system. Note that the action of the Geroch group would be trivial if we were starting from flat space in Cartesian coordinates because there are no other physical solutions of the Ernst $\sigma$ model with $\operatorname{det} g= \pm 1$ that are also compatible with the equations for the conformal factor $f$. Actually, the conformal factor has a very special role incorporating the effects of 2D gravity in the reduced form of the string background equations.
For certain discrete values of the soliton moduli the underlying string backgrounds are equivalent as exact conformal field theories, as it was demonstrated explicitly for the general one-parameter form of the Nappi-Witten universe. This happens because the corresponding $\mathrm{O}(2,2 ; Z)$ $T$-duality transformations [17] act on the soliton moduli as described. Since the soliton transformations provide specific elements of the string Geroch group, i.e., the current group $\hat{\mathrm{O}}(2,2)$ in the simplest case under discussion, and they also contain $S$ transformations and their intertwining with $T$, our description suggests that other discrete remnants of this infinite-dimensional group could act as $U$ dualities in string compactifications to two dimensions $[4,10]$.

Further progress in this direction certainly requires extending the formalism to include gauge fields as well. For example, it will be interesting to describe 4D black holes with electric charge $Q$ as 2D solitons of an Ernst-type $\mathrm{SU}(2,1) \sigma$ model. The points in the soliton moduli space that describe extremal black holes are special in that a solitonic interpretation of the resulting configuration exists in four as well as in two (reduced) dimensions. This should be ultimately extended to $\mathrm{O}(8,24) \sigma$ models, which are applicable to heterotic string compactifications to two dimensions $[10,11]$. One should also try to find in this context the necessary conditions on the Killing isometries so that the solitonic nature of a given configuration is preserved or attained under reduction. It seems that the relevant distinction in the examples we have considered so far is provided by the translational vs the rotational character of the corresponding Killing vector fields (using the same terminology as in [3]). For Euclidean black holes, for example, $\partial / \partial t$ is rotational unless $M=B$ (self-dual Taub-NUT limit with $A=0$ in the vacuum case) or $M=Q$ (extremal nonrotating solution of the electrovacuum equations), in which cases we find that $\partial / \partial t$ becomes translational. These two examples demonstrate clearly that the reduction with respect to rotational isometries can give rise to $2 \mathrm{D}$ solitonic configurations, in the sense described above, even if the 4D configurations are not so. A characteristic feature of rotational isometries in supersymmetric backgrounds is that the fermions depend on the Killing coordinates, while the bosonic fields do not, and hence it is such as having a coordinate-dependent compactification.

The soliton solutions of the 4D theory (Bogomolny, Prasad, Sommerfield states) are quantum-mechanically stable having manifest space time supersymmetry. On the other hand, the 2D solitonic interpretation of a given configuration was only used here in the context of the inversescattering method with no reference to supersymmetry. Hence, a natural question arises to find the special properties of these $2 \mathrm{D}$ solitons with respect to $2 \mathrm{D}$ reductions of the spacetime supersymmetry algebra. To the best of our knowledge the only helpful results that exist in the literature are contained in [22], where the 2-dim reduction of maximal supergravity (and some of its consistent truncations) were described in terms of Lax pairs and integrable structures. We briefly mention that the local supersymmetry transformations can be bosonized in two dimensions and they can be included as Kac-Moody variations into the corresponding infinite-dimensional hidden symmetry (Geroch-type) transformations. Also, the variation of the conformal factor that accounts for the 2D gravitational effects in this case can be 
included systematically using central extensions of the associated current groups [23]. Further work is certainly required in this direction, putting together in a more constructive way the vast variety of soliton solutions obtained by the inversescattering methods with the supersymmetric configurations of the $2 \mathrm{D}$ reduced supergravity. It is conceivable that $11 \mathrm{D}$ supergravity will be singled out in this line of thought, according to earlier expectations by Nicolai [22].

Summarizing, the 2D reduced sector of string theory is quite rich in symmetry due to the integrability structure of the lowest order effective theory. Better understanding of its soliton solutions are worthy in the light of the recent developments in nonperturbative string theory, in order to establish and explore the meaning of infinitely many $U$ dualities in the spectrum. This particular sector of string theory, where many more connections are expected to exist than those in other sectors, could also help in the long run to expose better the right structures that are needed for the ultimate formulation of string theory. Could it be an exactly solvable sector? We hope to return to it elsewhere.
[1] C. Hull and P. Townsend, Nucl. Phys. B438, 109 (1995).

[2] E. Witten, Nucl. Phys. B443, 85 (1995).

[3] I. Bakas, Phys. Lett. B 343, 103 (1995).

[4] I. Bakas, Nucl. Phys. B428, 374 (1994).

[5] R. Geroch, J. Math. Phys. (N.Y.) 12, 918 (1971); 13, 394 (1972).

[6] E. Cremmer and B. Julia, Nucl. Phys. B159, 141 (1979); B. Julia, in Superspace and Supergravity, edited by S. Hawking and M. Rocek (Cambridge University Press, Cambridge, England, 1981).

[7] N. Marcus and J. Schwarz, Nucl. Phys. B228, 145 (1983).

[8] H. Nicolai, in Recent Aspects of Quantum Fields, Proceedings of 30th Schladming Winter School, Schladming, Austria, 1991, edited by H. Mitter and H. Gausterer, Lecture Notes in Physics Vol. 396 (Springer, Berlin, 1991).

[9] A. Sen, Nucl. Phys. B434, 179 (1995).

[10] A. Sen, Nucl. Phys. B447, 62 (1995).

[11] J. Schwarz, Nucl. Phys. B454, 427 (1995).

[12] C. Nappi and E. Witten, Phys. Lett. B 293, 309 (1992).
[13] C. Kounnas and D. Lust, Phys. Lett. B 289, 56 (1992).

[14] C. Callan, J. Harvey, and A. Strominger, Nucl. Phys. B359, 611 (1991)

[15] C. Kounnas, Phys. Lett. B 321, 26 (1994).

[16] I. Antoniadis, S. Ferrara, and C. Kounnas, Nucl. Phys. B421, 343 (1994).

[17] A. Giveon and A. Pasquinucci, Phys. Lett. B 294, 162 (1992).

[18] M. Rocek, K. Schoutens, and A. Sevrin, Phys. Lett. B 265, 303 (1991).

[19] V. Belinski and V. Sakharov, Sov. Phys. JETP 48, 985 (1978); 50, 1 (1979).

[20] V. Ferrari, J. Ibanez, and M. Bruni, Phys. Rev. D 36, 1053 (1987); V. Ferrari and J. Ibanez, Gen. Relativ. Gravit. 19, 405 (1987).

[21] C. Cosgrove, J. Math. Phys. (N.Y.) 21, 2417 (1980).

[22] H. Nicolai, Phys. Lett. B 194, 402 (1987); H. Nicolai and N. Warner, Commun. Math. Phys. 125, 369 (1989).

[23] P. Breitenlohner and D. Maison, Ann. Inst. Henri Poincaré Phys. Theor. 46, 215 (1987). 